\title{
An Analytic Platform for the Rapid and Reproducible Annotation of Ventilator Waveform Data
}

\section{A Platform for Annotating Ventilator Waveform Data}

Gregory B. Rehm ${ }^{1}$ MS*, Brooks T. Kuhn ${ }^{2}$ MD*, Monica K. Lieng ${ }^{3}$ BS, Irene Cortes-Puch ${ }^{2}$ MD, Jimmy Nguyen ${ }^{6}$ RRT-ACCS, Edward C. Guo ${ }^{1}$ BS, Jean-Pierre Delplanque ${ }^{4} \mathrm{PhD}$, Nicholas R.

\author{
Anderson ${ }^{5} \mathrm{PhD}$, Jason Y. Adams ${ }^{2} \mathrm{MD}$ \\ * These authors contributed equally to this work
}

Corresponding Author: Gregory B. Rehm: grehm@ucdavis.edu

${ }^{1}$ Department of Computer Science, University of California Davis

${ }^{2}$ Division of Pulmonary, Critical Care, and Sleep Medicine, University of California Davis

${ }^{3}$ School of Medicine, University of California Davis

${ }^{4}$ Department of Engineering, University of California Davis

${ }^{5}$ Department of Public Health Sciences, University of California Davis

${ }^{6}$ Division of Respiratory Care, University of California Davis 


\begin{abstract}
Algorithmic classifiers are crucial components of clinical decision support (CDS) systems needed to advance healthcare delivery. Robust CDS systems must be derived and validated via creation of multi-reviewer adjudicated gold standard datasets. Manual annotation of physiologic data such as mechanical ventilator waveform data (VWD) can be time-consuming, and lacks methodological consistency in dataset development. To address these issues, we have created a system for annotating and adjudicating VWD called the Annotation PipeLine (APL) to optimize VWD annotation by expert reviewers. APL combines visual assessment of waveform characteristics with metadata display, enabling inclusion of quantitative thresholds into annotation decisions by reviewers. APL also includes specific features for resolving multireviewer disagreements and generating gold standard data sets. APL's unique combination of methods and open source framework may accelerate the creation of CDS algorithms for ventilator management, and may serve as a model for future research into physiologic waveform annotation systems.
\end{abstract}

\title{
I. Introduction
}

Algorithmic classifiers promise to play a central role in analysis of different types of quantitative healthcare data, such as high-volume streaming waveform data gathered from physiologic monitoring devices. By utilizing expert-derived rules and machine learning, algorithmic classifiers can support CDS systems that can function with complex data types, and patient and disease heterogeneity. High-performance model development requires well characterized datasets annotated for salient events by expert clinicians through a process of multi-reviewer adjudication.(1-3) Accurately and reproducibly annotating data requires 
substantial investment of effort from time-limited, expert reviewers, which to date has limited the size and generalizability of validated datasets.(4-7)

These challenges are salient in research related to mechanical ventilation (MV). Incorporating algorithmic classifiers to MV practice has the potential to detect diagnostic events, pathologic phenomena such as patient-ventilator asynchronies (PVA), and improve clinical outcomes.(8-16). To develop and validate high performance classifiers, clinicians have historically been required to manually annotate large volumes of ventilator waveform data (VWD) by visually analyzing the shape and magnitude of air flow and pressure data. $(7-10,13)$ However, this process is time-consuming, subjective, and has been marked by low inter-rater agreement due to visual classification criteria based on "expert opinion", as well as data management challenges resulting from the high volume of data and the laborious manual annotation process. $(7,10,17)$ Previous groups have developed waveform annotation software that can handle large volume data and output categorization results, but these efforts lack the specific functionality necessary for overcoming problems inherent with annotating VWD.(18-30). These challenges are: 1) consistent annotation workflows do not exist for VWD annotation, which has led to the creation of datasets that are potentially non-reproducible across medical centers (17), and 2) large, high-quality, multi-reviewer adjudicated datasets of VWD are highly timeconsuming to generate for expert clinicians.(7)

With these challenges in mind, we sought to address gaps in the field of VWD annotation by developing the Annotation PipeLine (APL). APL supports expert annotation of ventilator data and builds on concepts from existing platforms for annotating data other than VWD. $(31,32)$ Utilizing APL, we have been able to annotate and provide dual clinician validation for over 315,000 ventilator recorded breaths across two different datasets. These data comprise some of 
the largest recorded breath-level, multi-clinician adjudicated datasets for use in developing algorithms to improve the delivery of MV.(1,7,9,10,13,33) APL's fundamental design features allow for consistent and efficient annotation of VWD, and may be broadly applied in future research to improve efficiency, reproducibility, and fidelity of medical waveform annotation and classifier development.

\section{Methods}

APL is a free, publicly accessible, web-based annotation platform developed since 2015 to support studies at University of California Davis (UCD) utilizing VWD collected from mechanically ventilated patients.(34) All VWD were collected under an approved IRB protocol and do not contain protected health information. Data were collected from Puritan Bennet Model $840^{\mathrm{TM}}$ (PB-840) ventilators (Covidien, Dublin, Republic of Ireland) and stored in multiple sequential files up to two hours in length. $(34,35)$

APL uses an open source stack including: ventMAP software for extraction of waveform metadata, the Python Flask web framework, the JavaScript library Dygraphs for graphing of VWD, Redis for storage, and Docker for APL deployment.(1,36,37) APL's source code and install instructions are available on GitHub for modification or use of APL.

\section{A. Improving Consistency of the Annotation Process}

APL was built to support objective classification of VWD by displaying clinicallyrelevant quantitative metadata to help improve the consistency of the annotation workflow. After data collection, VWD is uploaded to APL in CSV format, is preprocessed for graphical display, and automatically split into individual breaths. Each breath is processed to generate clinically relevant metadata pertinent to the classification of VWD using ventMAP (Figure 1).(1,38) Examples of metadata generated include the inspiratory tidal volume (the volume delivered by 
the ventilator with each breath) and the expiratory time (the duration of each exhalation). All derived metadata were determined to be clinically relevant by four critical care clinicians (JYA, BTK, ICP, JN). Metadata are cached in a CSV file and then retrieved by Flask to generate a breath-level drop-up table that displays breath-specific metadata and annotation options. Display of metadata in a drop-up table mimics the user interface of a mechanical ventilator and was designed to facilitate use of APL by expert reviewers (Figure 2).

Figure 1: Here we display the flow of data within APL as it is processed by our software architecture. Raw VWD is uploaded to APL and then processed for graphical rendering, using ventMAP metadata processing to extract clinical metadata from each breath. Results from processing are stored on the filesystem. Next, we visualize both VWD, and perform breath-level annotation by utilizing Dygraphs with our own custom annotation overlay. All annotations are stored in Redis, and can be output by the user into CSV format.

Figure 2: We designed APL to be familiar to clinicians, rendering information analagous to the user interface screen of a mechanical ventilator. Left, an image of the graphical user interface of a mechanical ventilator. Right, APL pressure (red) and flow (blue) waveforms with customizable breath-level metadata and point-click annotation. Breath interpretation at the bedside relies on both waveform and metadata analysis. APL presents both waveform morphology and breathlevel metadata for identification of threshold-defined events.

APL also incorporates functionality to support online reconciliation of discordant annotations to improve consistency and reproducibility of the annotation process. This functionality is possible because APL is an online web platform and can store all user annotations centrally in the Redis database. Stored annotations can then be retrieved later by the Python Flask web framework to compare classification decisions by different reviewers. Differing classifications are displayed by Dygraphs as a red label that contains the cause for classification disagreement. Dygraphs displays a green checkbox above a breath if no disagreement for a breath is found.

The annotation view (AV) functionality provides the ability to select subsets of available metadata and breath classifications to display when reviewers are performing annotation. AVs are aimed at reducing information overload for the user and simplifying the annotation process 
by targeting it to specific project requirements. All AVs are stored in Redis, and new AVs can be created by a user by selecting the specific metadata and breath classification desired.

\section{B. Improving Speed of the Annotation Process}

To streamline the end-to-end annotation workflow, we designed a unified system of annotation and visual display termed 'on-screen annotation' that allows for simultaneous display of waveform morphology, metadata, and annotation choices for every breath. (Figure 1b) Onscreen annotation is accomplished by coding customized instructions in JavaScript that render drop-up tables containing metadata, annotation choices, and server callback logic. We then ensure that Dygraphs runs our overlay software after rendering of VWD. Using this overlay interface, we incorporated additional functionality to improve the speed of how annotations are performed, such as the option to perform single or multi-breath classifications.

\section{Results}

Before starting the annotation process, users typically visit the Settings page to choose an AV (Figure 3). While users may choose a pre-defined AV, project leaders can create an AV, requiring that all other reviewers utilize that view to enforce consistency of annotations and outputs for a dataset. When an AV is created, leaders choose from a variety of annotation options and metadata available for display (Figure 3). When finished, the view can be named and saved to be used by other project members. In our initial work, we utilized AVs to create two different gold-standard datasets for PVA and ventilator mode (VM) classification.

Figure 3: We display a typical workflow for a user when using APL, and screenshots from APL that display typical uses and functionality. On the left, annotation workflow discusses the individual steps a user would take to annotate a file. On the right three images are shown: the first showing how a user would create a new annotation view, the second displays how the annotation process occurs on ventilator waveform data. The third shows how reconciliation occurs with discordant classifications highlighted in red, displaying exactly which classifications are mismatched. Agreements are displayed as a checkbox. 
Once a view is selected, uploaded VWD files can be graphically displayed for annotation. If reviewers only wish to annotate portions of a file, they can zoom in and out of specific breath regions using Dygraphs to focus on regions of interest. When a specific breath is annotated, the user can click on its breath number, and a drop-up window will appear. Drop-up windows display both quantitative metadata and annotation options allowed by the current AV (Figure 2). Users can annotate a breath by selecting the checkbox corresponding to the specific annotation desired. A user can also annotate multiple consecutive breaths using multi-breath functionality where the user annotates the first and the final breaths in a series, and all intermediate annotations will be automatically completed.

After multiple users annotate the same file, reconciliation of discordant annotations can occur. Reconciliation functionality displays VWD using an interface similar to annotation, but highlights disagreements in red and agreements with green checkboxes. Reviewers can evaluate each disagreement simultaneously and collaborate to resolve them. Once finished, users can output a CSV file containing all gold standard breath-level annotations.

Using APL, three critical care clinicians from UCD (BTK, JN, and JYA) annotated breaths for 18 different classifications from 254 VWD patient files, and generated two datasets totaling 318,676 multi-clinician adjudicated breaths. To our knowledge, this collection represents some of the largest reported datasets of breath-level, multi-clinician adjudicated VWD (Table 1).

\begin{tabular}{|l|r|r|}
\hline Dataset & $\begin{array}{l}\text { Number of } \\
\text { Annotated Breaths }\end{array}$ & $\begin{array}{l}\text { Number of Breath } \\
\text { Classifications }\end{array}$ \\
\hline Pohlman 2008 (39) & 391 & 2 \\
\hline Blanch 2012 (40) & 1,024 & 2 \\
\hline Gholami 2018 (41) (using ExpertLabeler) & 1,377 & 3 \\
\hline Sottile 2018(7) & 2,500 & 4 \\
\hline Colombo 2011 (15,17) & 3,731 & 4 \\
\hline
\end{tabular}




\begin{tabular}{|l|r|r|}
\hline Adams 2017 (1) (using APL) & 9,719 & 8 \\
\hline Thille 2006 (42) & $35,580-58,110$ & 3 \\
\hline Beitler 2016 (10) & 143,199 & 1 \\
\hline Ventilator Mode (using APL; in submission) & 308,957 & 10 \\
\hline
\end{tabular}

Table 1: Existing multi-clinician adjudicated datasets of VWD. Number of classes corresponds with the number of annotation decisions available to the clinician as they performed annotation, including normal breaths. Exact counts for number of breaths annotated by Thille were not mentioned in the literature, so a range was extrapolated from the number of patients enrolled in their study and the respiratory rate of patients.

\section{Discussion}

Creating datasets of large, multi-clinician annotated VWD for MV research is limited by a lack of accessible, efficient, and reproducible multi-reviewer annotation methods. We developed the Annotation PipeLine (APL) to remedy these issues for VWD annotation and perform structured output of results. APL uses breath metadata to create hybrid visualquantitative classifications of VWD. Incorporation of quantitative and qualitative methods into a web-based visual annotation interface may reduce reviewer subjectivity in VWD classification, and enables efficient resolution of reviewer disagreements to generate gold standard data sets to drive classifier development. Using these methods, our team has annotated 318,676 dualadjudicated breaths for use in development of VM and PVA detection algorithms, which represents one of the largest existing collections of multi-reviewer annotated VWD. $(1,7,15,17,39,40)$

Historically, VWD annotation has been performed either by hand, use of electronic spreadsheets, and most recently by the web-based ExpertLabeler system.(41) A major limitation of these methods is that classifications are performed by visual criteria alone which are subject to high inter-rater bias due to reviewer subjectivity and local practice pattern.(17) APL addresses this limitation with the development of methods to support the simultaneous use of waveform morphology and metadata derived from VWD in classification decisions. To our knowledge, no 
MV-specific annotation package exists that is able to compute breath level metadata, display it on-screen, and allow multi-reviewer annotation and adjudication of events.

Technologically, APL builds on previous work related to annotation platform development and features novel contributions of its own. Our work to display VWD builds on previous platforms that provide annotation of generic physiologic data such as AcqKnowledge, ChronoViz, and BEDA, which have exemplified how to perform annotation on temporally archived waveform data.(18,20,43-45) APL's combined morphologic and metadata display functionality also builds upon several previous studies that have derived higher-level metadata from raw input streams for purposes of display and annotation.(26,46-49) For example, Jing et al developed the ability to annotate multiple electroencephalogram (EEG) waveforms simultaneously.(50) Similarly, APL extends existing work developed in natural language processing applications for inter-rater reconciliation to a new domain of streaming physiologic waveform annotation. $(31,32)$ Finally, APL contributes novel functionality including the use of drop-up windows with combined metadata and annotation capabilities, and the addition of annotation views that enable different metadata and annotation capabilities to be enforced across teams and customized to specific research needs.

Our work is limited by use of data and clinicians from a single center, and APL is presently limited to processing VWD from a single ventilator. Future work will generalize APL's data processing capabilities to other ventilators and medical devices by exploring the use of emerging waveform encoding standards.(51) In addition, APL has only been used by four clinicians to date and further modifications may be required to meet the needs of other researchers. Changes to APL may also enable additional investigation into the reproducibility of waveform classification, and may facilitate the development of consensus guidelines for 
annotating VWD.(17,52,53) To promote further research, we have freely released our code on GitHub so that the wider research community may modify our work.

Creating effective algorithms that can detect the presence of common diseases and pathologic respiratory phenomena for CDS will require large, gold-standard datasets of VWD. In order to create generalizable datasets, researchers will also need consensus definitions for MVrelated phenomena. To satisfy these needs we developed APL, the first open-source, multireviewer MV visualization and annotation platform that allows for the use of multi-epoch annotation to speed the classification process, customizable annotation views to constrain and standardize class assignments, reconciliation of inter-rater classification disagreements, and the simultaneous display of waveform morphology and breath level metadata to reduce classification subjectivity. While APL is specific to MV, the concepts APL utilizes can be applied to other types of high volume, physiologic waveform data. In summary, APL represents a new, effective tool to improve the consistency and speed of generating multi-reviewer gold-standard VWD datasets that will help drive the development of novel CDS systems.

\section{REFERENCES}

1. Adams JY, Lieng MK, Kuhn BT, Rehm GB, Guo EC, Taylor SL, et al. Development and Validation of a Multi-Algorithm Analytic Platform to Detect Off-Target Mechanical Ventilation. Sci Rep. Nature Publishing Group; 2017 Nov 3;7(1):14980.

2. Calvert JS, Price DA, Chettipally UK, Barton CW, Feldman MD, Hoffman JL, et al. A computational approach to early sepsis detection. Computers in Biology and Medicine. Pergamon; 2016 Jul 1;74:69-73.

3. Yao L, Poblenz E, Dagunts D, Ben Covington, Bernard D, Lyman K. Learning to diagnose from scratch by exploiting dependencies among labels. Vol. cs.CV, arXiv.org. 2017.

4. Tomlinson DR, Bashir Y, Betts TR, Rajappan K. Accuracy of manual QRS duration assessment: its importance in patient selection for cardiac resynchronization and implantable cardioverter defibrillator therapy. Europace. Oxford University Press; 2009 May 1;11(5):638-42. 
5. Baumgartner WA, Cohen KB, Fox LM, Acquaah-Mensah G, Hunter L. Manual curation is not sufficient for annotation of genomic databases. Bioinformatics. 2nd ed. Oxford University Press; 2007 Jul 1;23(13):i41-8.

6. Bird C, Bachmann A, Rahman F, Bernstein A. LINKSTER: enabling efficient manual inspection and annotation of mined data. New York, New York, USA: ACM; 2010. pp. 369-70.

7. Sottile PD, Albers D, Higgins C, Mckeehan J, Moss MM. The Association Between Ventilator Dyssynchrony, Delivered Tidal Volume, and Sedation Using a Novel Automated Ventilator Dyssynchrony Detection Algorithm*. Critical Care Medicine. Wolters Kluwer; 2018 Feb;46(2):e151-7.

8. Blanch L, Villagra A, Sales B, Montanya J, Lucangelo U, Luján M, et al. Asynchronies during mechanical ventilation are associated with mortality. Intensive Care Med. 2015 Feb 19;41(4):633-41.

9. Chanques G, Kress JP, Pohlman A, Patel S, Poston J, Jaber S, et al. Impact of Ventilator Adjustment and Sedation-Analgesia Practices on Severe Asynchrony in Patients Ventilated in Assist-Control Mode*. Critical Care Medicine. 2013 Sep;41(9):2177-87.

10. Beitler JR, Sands SA, Loring SH, Owens RL, Malhotra A, Spragg RG, et al. Quantifying unintended exposure to high tidal volumes from breath stacking dyssynchrony in ARDS: the BREATHE criteria. Intensive Care Med. Springer Berlin Heidelberg; 2016 Jun 24;42(9):1427-36.

11. Acute Respiratory Distress Syndrome Network, Brower RG, Matthay MA, Morris A, Schoenfeld D, Thompson BT, et al. Ventilation with lower tidal volumes as compared with traditional tidal volumes for acute lung injury and the acute respiratory distress syndrome. N Engl J Med. 2000 May 4;342(18):1301-8.

12. van Drunen EJ, Chiew Y, Chase J, Shaw GM, Lambermont B, Janssen N, et al. Expiratory model-based method to monitor ARDS disease state. BioMedical Engineering OnLine 2014 13:1. BioMed Central; 2013;12(1):57.

13. Mulqueeny Q, Ceriana P, Carlucci A, Fanfulla F, Delmastro M, Nava S. Automatic detection of ineffective triggering and double triggering during mechanical ventilation. Intensive Care Med. 1st ed. Springer-Verlag; 2007;33(11):2014-8.

14. Koenig HC, Finkel BB, Khalsa SS, Lanken PN, Prasad M, Urbani R, et al. Performance of an automated electronic acute lung injury screening system in intensive care unit patients*. Critical Care Medicine. 2011 Jan 1;39(1):98-104.

15. Sinderby C, Liu S, Colombo D, Camarotta G, Slutsky AS, Navalesi P, et al. An automated and standardized neural index to quantify patient-ventilator interaction. Critical Care. BioMed Central; 2013;17(5):R239.

16. Piquilloud L, Jolliet P, Revelly J-P. Automated detection of patient-ventilator asynchrony: 
new tool or new toy? Critical Care. BioMed Central; 2013;17(6):1015.

17. Colombo D, Cammarota G, Alemani M, Carenzo L, Barra FL, Vaschetto R, et al. Efficacy of ventilator waveforms observation in detecting patient-ventilator asynchrony. Critical Care Medicine. 2011 Nov;39(11):2452-7.

18. Kim J, Snodgrass M, Pietrowicz M. BEDA: Visual analytics for behavioral and physiological data. 2013. pp. 23-7.

19. Gramfort A, Luessi M, Larson E, Engemann DA, Strohmeier D, Brodbeck C, et al. MNE software for processing MEG and EEG data. NeuroImage. Academic Press; 2014 Feb $1 ; 86: 446-60$.

20. Fouse A, Weibel N, Hutchins E, Hollan JD. ChronoViz: a system for supporting navigation of time-coded data. Proceedings of the 2011 annual conference extended abstracts on Human factors in computing systems - CHI EA '11. ACM; 2011. 6 p.

21. Sousa EAF, Malheiro TEQ, Bicho E, Erlhagen W, Santos JA, Pereira AF. MUVTIME: a Multivariate time series visualizer for behavioral science. 11th Joint Conference on Computer Vision, Imaging and Computer Graphics Theory and Applications. SCITEPRESS - Science and and Technology Publications; 2016 Feb 1;:165-76.

22. Pandey S, Voorsluys W, Niu S, Khandoker A, Buyya R. An autonomic cloud environment for hosting ECG data analysis services. Future Generation Computer Systems. NorthHolland; 2012 Jan 1;28(1):147-54.

23. Stockbridge N, Brown BD. Annotated ECG waveform data at FDA. Journal of Electrocardiology. 2004;37:63.

24. Brugman H, Russel A. Annotating Multi-media/Multi-modal Resources with ELAN. Corpus technology and language pedagogy: New resources, new tools, new methods. 2004 May 26.

25. Müller C, Strube M. Multi-level annotation of linguistic data with MMAX2. Corpus technology and language pedagogy: New resources, new tools, new methods. 2006;3:197214.

26. Winslow RL, Granite S, Jurado C. WaveformECG: A Platform for Visualizing, Annotating, and Analyzing ECG Data. IEEE Transactions on Medical Imaging. 2016 Sep $18 ; 18(5): 36-46$.

27. Russell BC, Torralba A, Murphy KP, Freeman WT. LabelMe: A Database and WebBased Tool for Image Annotation. Int J Comput Vis. Springer US; 2007 Oct 31;77(13):157-73.

28. Götz S, García-Gómez JM, Terol J, Williams TD, Nagaraj SH, Nueda MJ, et al. Highthroughput functional annotation and data mining with the Blast2GO suite. Nucleic Acids Res. Oxford University Press; 2008 Jun 1;36(10):3420-35. 
29. Letunic I, Bork P. Interactive Tree Of Life v2: online annotation and display of phylogenetic trees made easy. Nucleic Acids Res. Oxford University Press; 2011 Jul 1;39(suppl_2):W475-8.

30. Ernst J, Kellis M. Large-scale imputation of epigenomic datasets for systematic annotation of diverse human tissues. Nature Biotechnology. Nature Publishing Group; 2015 Apr 1;33(4):364-76.

31. Stan J, Demner-Fushman D, Fung KW, Bodenreider O. Facilitating reconciliation of interannotator disagreements. AMIA Annu Symp Proc. 2014.

32. Kilicoglu H, Demner-Fushman D. Bio-SCoRes: A Smorgasbord Architecture for Coreference Resolution in Biomedical Text. Groza T, editor. PLOS ONE. Public Library of Science; 2016 Mar 2;11(3):e0148538.

33. Murias G, Montanya J, Chacón E, Estruga A, Subirà C, Fernández R, et al. Automatic detection of ventilatory modes during invasive mechanical ventilation. BioMed Central; 2016 Aug 14;20(1):258.

34. Rehm GB, Kuhn BT, Delplanque J-P, Guo EC, Lieng MK, Nguyen J, et al. Development of a research-oriented system for collecting mechanical ventilator waveform data. J Am Med Inform Assoc. 2017 Oct 28;25(3):295-9.

35. Covidien. Operator's and Technical Reference Manual. Puritan Bennett 800 Series Ventilator System. Covidien; 2011.

36. Merkel D. Docker: lightweight Linux containers for consistent development and deployment. Linux Journal. Belltown Media; 2014 Mar 1;2014(239):2.

37. Vanderkam D. Dygraphs Javascript Charting Library. 2006.

38. Rehm G, Han J, Kuhn B, Delplanque J-P, Anderson N, Adams J, et al. Creation of a Robust and Generalizable Machine Learning Classifier for Patient Ventilator Asynchrony. Methods Inf Med. 2018 Sep 24;57(04):208-19.

39. Pohlman MC, McCallister KE, Schweickert WD, Pohlman AS, Nigos CP, Krishnan JA, et al. Excessive tidal volume from breath stacking during lung-protective ventilation for acute lung injury*. Critical Care Medicine. 2008 Nov 1;36(11):3019-23.

40. Blanch L, Sales B, Montanya J, Lucangelo U, García-Esquirol O, Villagra A, et al. Validation of the Better Care ${ }^{\circledR}$ system to detect ineffective efforts during expiration in mechanically ventilated patients: a pilot study. Intensive Care Med. Springer-Verlag; 2012;38(5):772-80.

41. Gholami B, Phan TS, Haddad WM, Cason A, Mullis J, Price L, et al. Replicating human expertise of mechanical ventilation waveform analysis in detecting patient-ventilator cycling asynchrony using machine learning. Computers in Biology and Medicine. Pergamon; 2018 Jun 1;97:137-44. 
42. Thille AW, Rodriguez P, Cabello B, Lellouche F, Brochard L. Patient-ventilator asynchrony during assisted mechanical ventilation. Intensive Care Med. Springer-Verlag; 2006;32(10):1515-22.

43. Hienert D, Wegener D, Schomisch S. Making Sense of Open Data Statistics with Information from Wikipedia. In: Availability, Reliability, and Security in Information Systems and HCI. Berlin, Heidelberg: Springer, Berlin, Heidelberg; 2013. pp. 329-44. (Lecture Notes in Computer Science; vol. 8127).

44. Wester M. AcqKnowledge, 1993, BIOPACK Systems. Inc: Goleta.

45. Benedek M, Kaernbach C. Decomposition of skin conductance data by means of nonnegative deconvolution. Psychophysiology. Blackwell Publishing Inc; 2010 Jul $1 ; 47(4): 647-58$.

46. Yu C, Zhong Y, Smith T, Park I, Huang W. Visual Data Mining of Multimedia Data for Social and Behavioral Studies. Information Visualization. SAGE PublicationsSage UK: London, England; 2009 Feb 12;8(1):56-70.

47. Ikaro Silva GBM. An Open-source Toolbox for Analysing and Processing PhysioNet Databases in MATLAB and Octave. Journal of open research software. NIH Public Access; 2014;2(1):E215.

48. Zisook M, Hernandez J, Goodwin MS. Enabling visual exploration of long-term physiological data. 2013.

49. Moody GB, Mark RG, Goldberger AL. PhysioNet: Physiologic signals, time series and related open source software for basic, clinical, and applied research. 2011.

50. Jing J, Dauwels J, Rakthanmanon T, Keogh E, Cash SS, Westover MB. Rapid annotation of interictal epileptiform discharges via template matching under Dynamic Time Warping. Journal of Neuroscience Methods. Elsevier; 2016 Dec 1;274:179-90.

51. Sauermann S, David V, Schlögl A, Egelkraut R, Frohner M, Pohn B, et al. Biosignals, Standards and FHIR - The Way to Go? Stud Health Technol Inform. 2017;236:356-62.

52. Kligfield P, Gettes LS, Bailey JJ, Childers R, Deal BJ, Hancock EW, et al. Recommendations for the Standardization and Interpretation of the Electrocardiogram: Part I: The Electrocardiogram and Its Technology A Scientific Statement From the American Heart Association Electrocardiography and Arrhythmias Committee, Council on Clinical Cardiology; the American College of Cardiology Foundation; and the Heart Rhythm Society Endorsed by the International Society for Computerized Electrocardiology. Journal of the American College of Cardiology. Journal of the American College of Cardiology; 2007 Mar 13;49(10):1109-27.

53. Mason JW, Hancock EW, Gettes LS. Recommendations for the Standardization and Interpretation of the Electrocardiogram: Part II: Electrocardiography Diagnostic Statement List A Scientific Statement From the American Heart Association Electrocardiography 
and Arrhythmias Committee, Council on Clinical Cardiology; the American College of Cardiology Foundation; and the Heart Rhythm Society Endorsed by the International Society for Computerized Electrocardiology. Journal of the American College of Cardiology. Journal of the American College of Cardiology; 2007 Mar 13;49(10):112835 . 
2017-01-01-01-01-01.1111

BS, S: 65426 ,

$3.14,11.41$

$9.49,11.55$

$24.14,12.56$

$39.42,14.47$

$51.89,17.23$

$59.10,20.62$

$59.21,24.22$

$50.34,26.61$

$42.50,27.35$

$40.52,27.77$

Ventilator Waveform Data

$\begin{array}{rrrrrrrr}\text { CO } & \text { dbL } & \text { fa } & \text { su } & \text { aNOS } & \text { mt } & \text { bs } & \text { WNOS } \\ 0 & 0 & 0 & 0 & 0 & 0 & 1 & 0 \\ 0 & 0 & 0 & 0 & 0 & 0 & 1 & 0 \\ 0 & 0 & 0 & 0 & 0 & 0 & 1 & 0 \\ 1 & 0 & 0 & 0 & 0 & 0 & 0 & 0 \\ 0 & 0 & 0 & 0 & 0 & 0 & 1 & 0 \\ 0 & 0 & 0 & 0 & 0 & 0 & 1 & 0\end{array}$

Annotations

DioRxIV preprint doI: https://dol.org/10.1101/568386; this v rsion posted March 6, 2019. The copyright holder for this preprint (which was not dertified by peer review) is the author/funder, who has gran ed bioRxiv a license to display the preprint in perp tuity. It is made available under

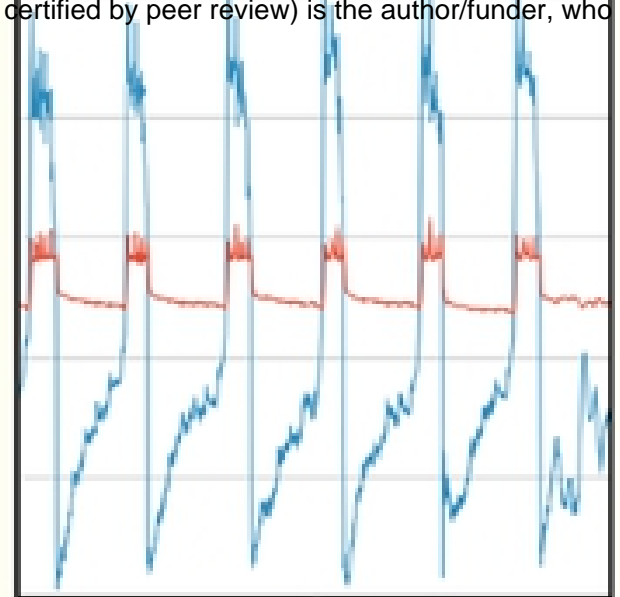

Graphical Processing

Metadata

Processing

Filesystem

Cache

Custom

Javascript Annotation

Interface

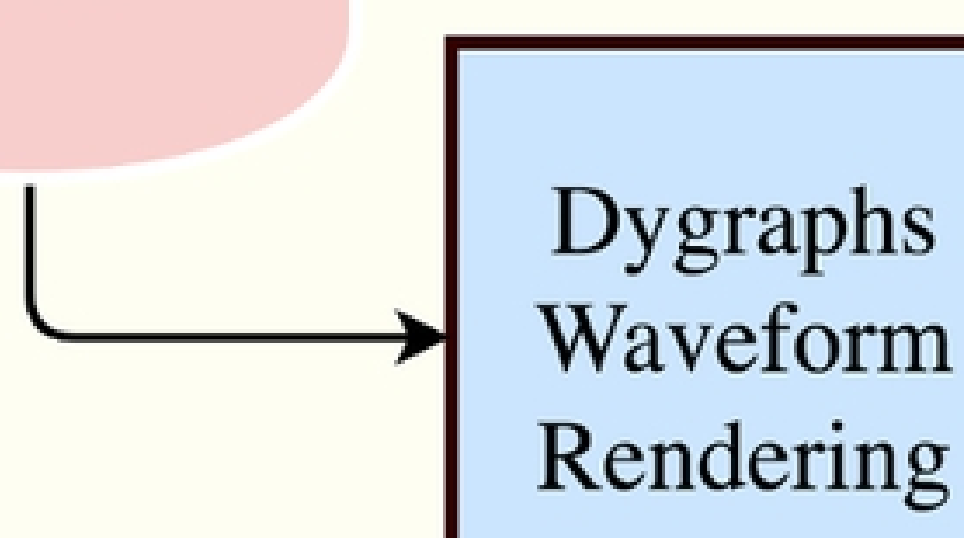

APL

Figure 1 


\section{Annotation Workflow}

\section{User Perspective}

Upload File

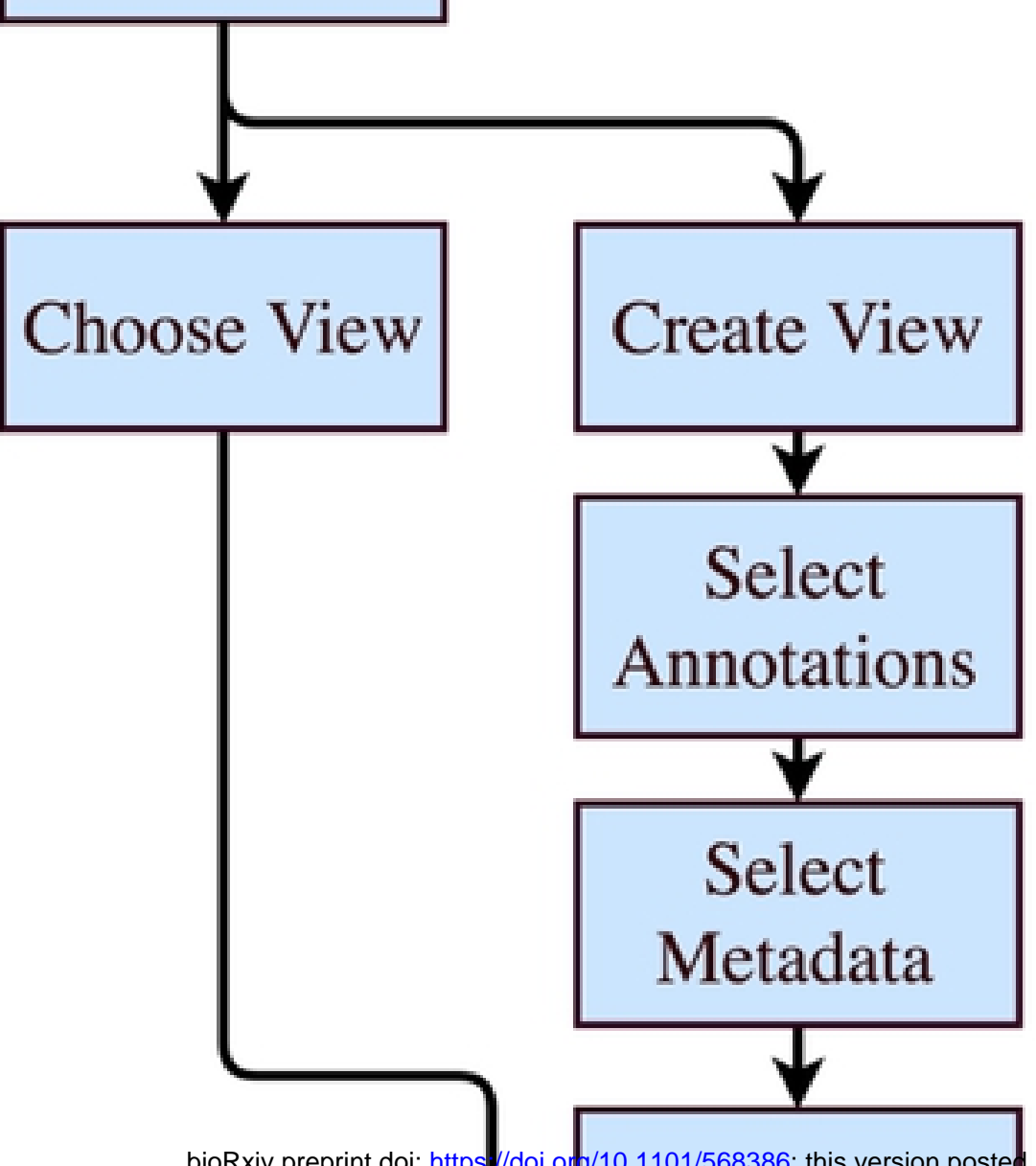

bioRxiv preprint doi: https//doi.ong/10.1101/568386; this version posteli March 6, 2019. The copyri
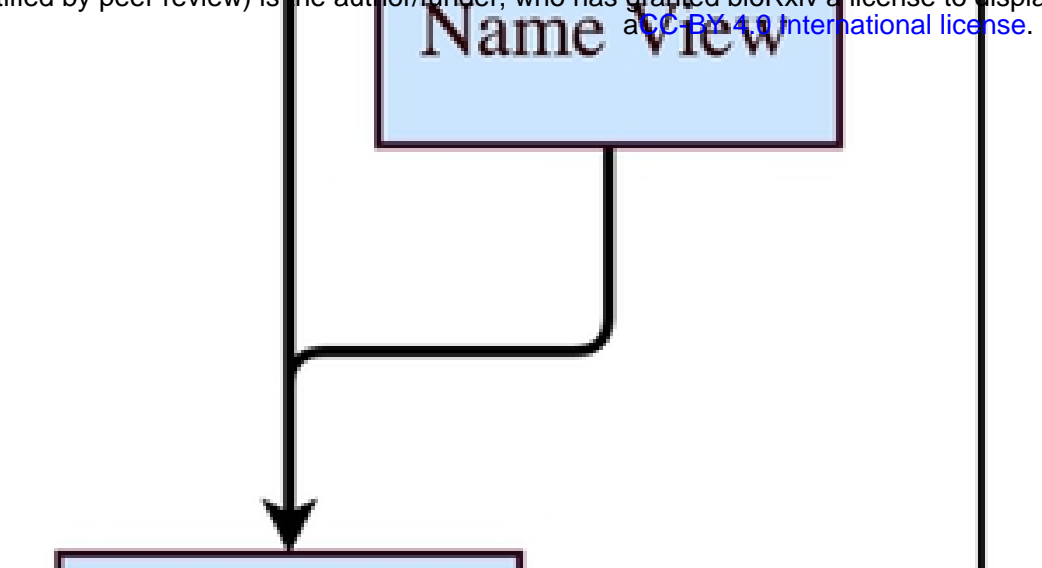

Visualize File
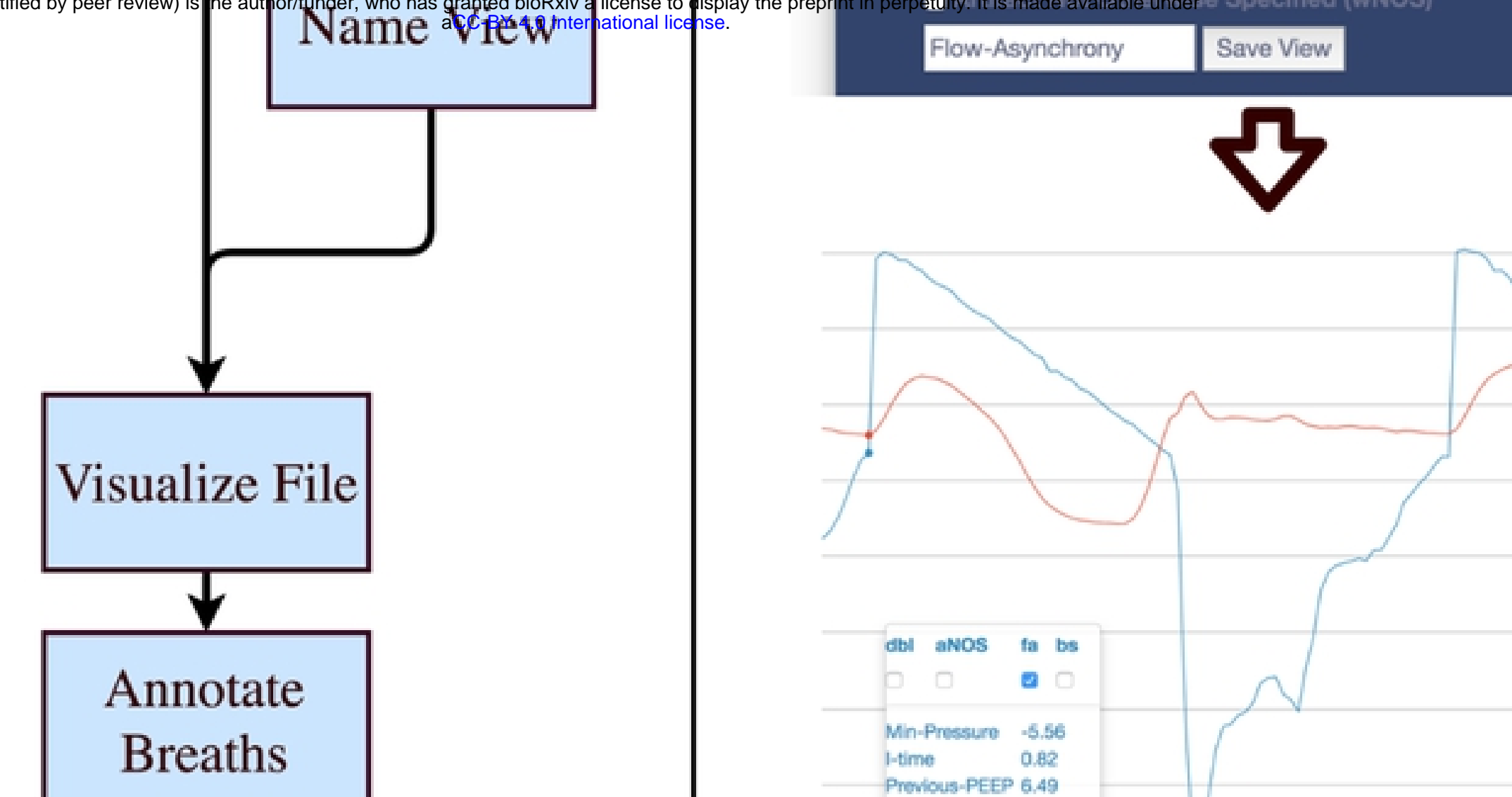

\section{CHOOSE VIEW NEW VIEW}

Data Type PVA Vent Mode

Metadata Options

Q Tidal Volume Air Exhaled (TVe)

Q Tidal Volume Air Inhaled (TV)

Q Ratio of TVe and TVi (TVe/TVi)

Q PEEP on Previous Breath (Provious-PEEP)

Q Positive End Expiratory Pressure (PEEP)

จ Minimum Pressure on Inspiration (Min-Pressure)

Q Inspiratory Time ((-time)

Q Expiratory Time (E-time)

D. Flow Asynchrony (fa)

$\varnothing$ Double Trigger (dbi)

$\nabla$ Breath Stacking (bs)

$\checkmark$ Asynchrony Not Otherwise Specified (aNOS)

- Cough (co)

- Suction (su)

Q Multi Triggor (mit)

\begin{tabular}{l|l} 
Flow-Asynchrony & Save View \\
\hline
\end{tabular}
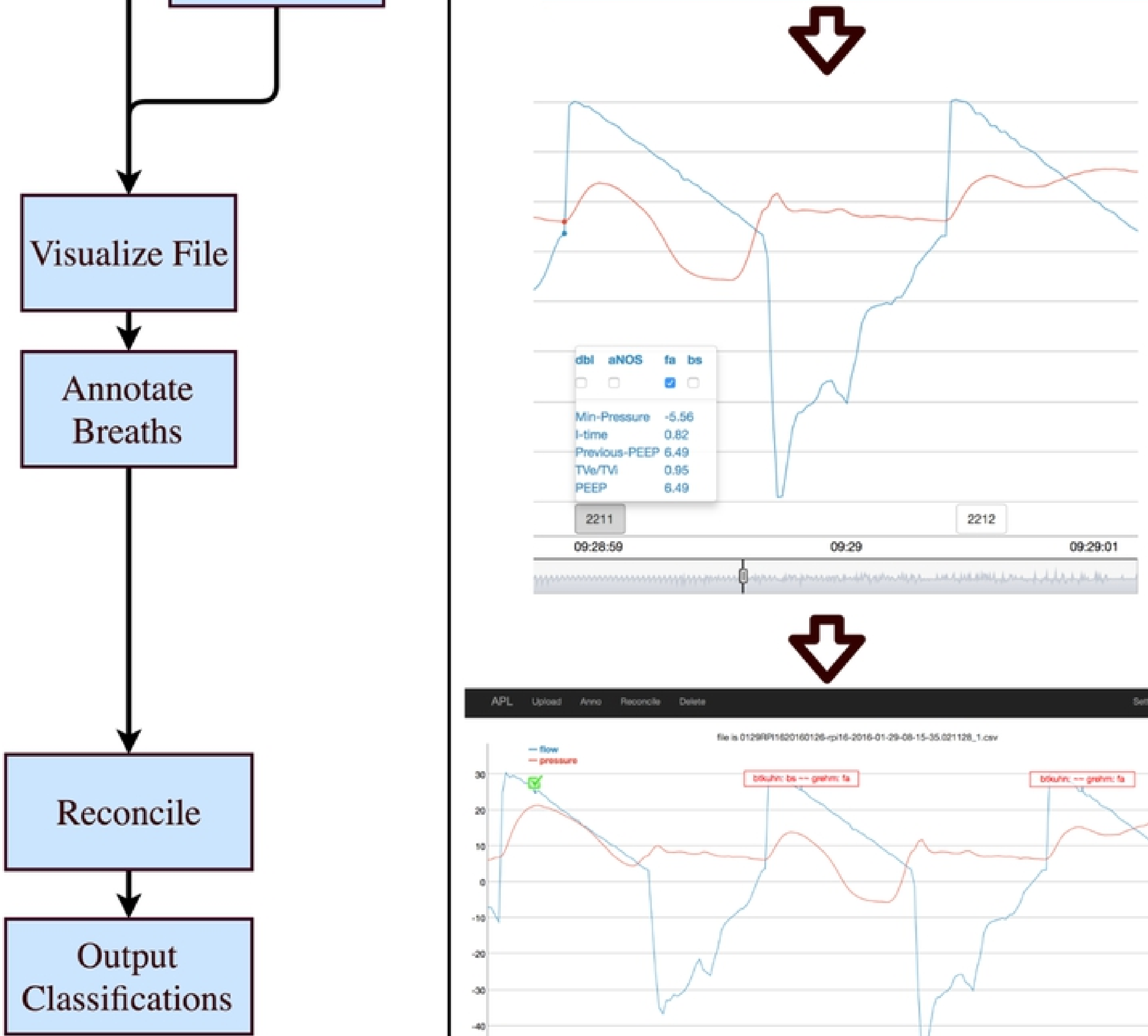

Figure 3

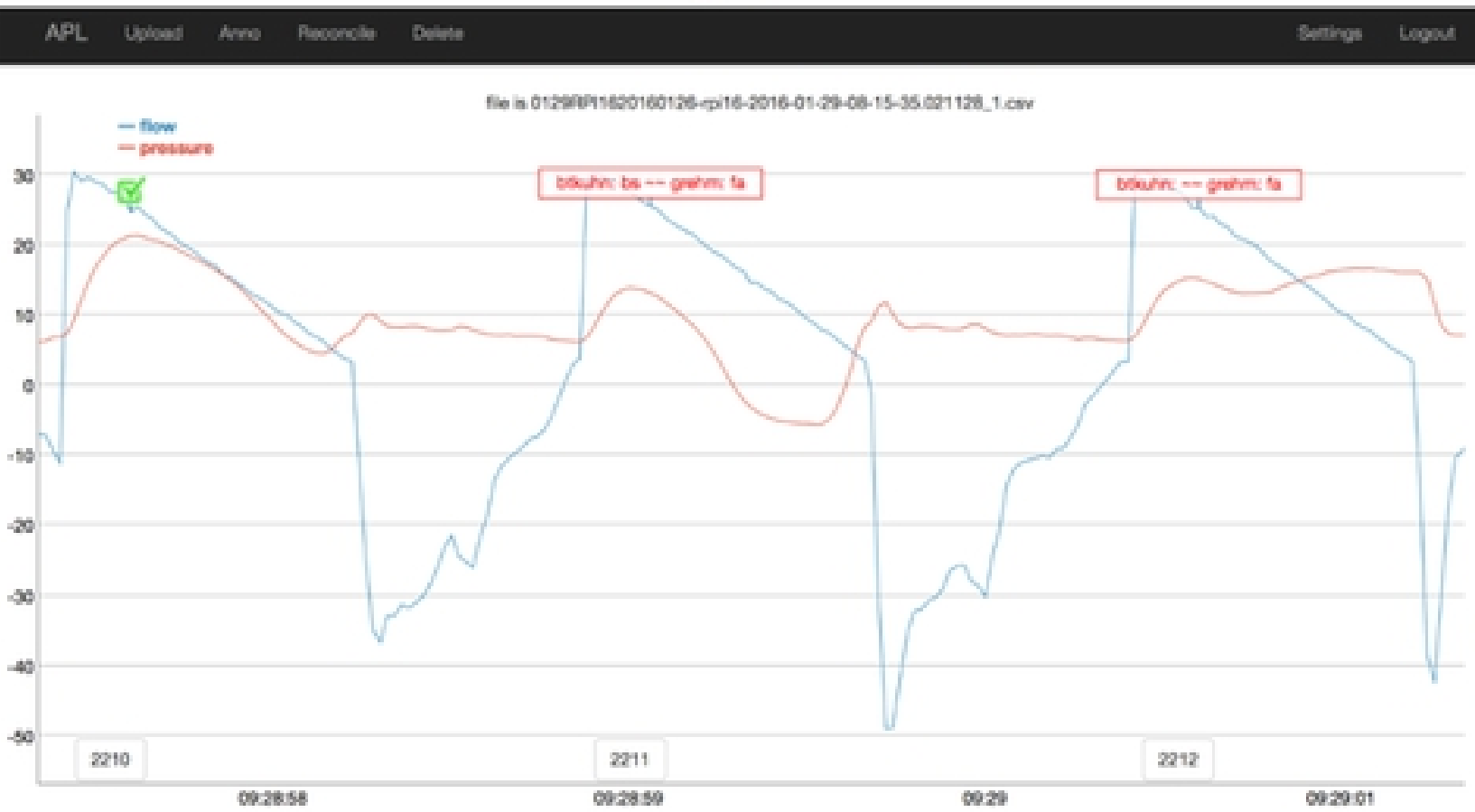

